\title{
Combination of lapatinib with isothiocyanates overcomes drug resistance and inhibits migration of HER2 positive breast cancer cells
}

\author{
Angelika Kaczyńska $^{1}$ - Anna Herman-Antosiewicz ${ }^{1}$
}

Received: 16 September 2015/ Accepted: 25 April 2016/Published online: 6 May 2016

(c) The Author(s) 2016. This article is published with open access at Springerlink.com

\begin{abstract}
Background Lapatinib is a commonly used drug that interrupts signaling from the epidermal growth factor receptors, EGFR and HER2/neu. Long-term exposure to lapatinib during therapy eliminates cells that are sensitive to the drug; however, at the same time it increases probability of lapatinib-resistant cell selection. The aim of this study was to verify whether combinations of lapatinib with one of isothiocyanates (sulforaphane, erucin or sulforaphene), targeting different levels of HER2 signaling pathway, exert stronger cytotoxic effect than therapy targeting the receptor only, using heterogeneous populations consisting of lapatinib-sensitive and lapatinib-resistant breast cancer cells.

Methods Lapatinib-sensitive HER2 overproducing SKBR-3 breast cancer cells and their lapatinib-resistant derivatives were combined at different proportions to simulate enrichment of cancer cell population in a drug-resistant fraction during lapatinib therapy. Effects of treatments on cell survival (MTT), apoptosis induction (PARP cleavage), prosurvival signaling (p-Akt, p-S6) as well as cell motility (wound healing assay) and invasion (Boyden chamber assay) were investigated.

Results Combination of lapatinib with any of isothiocyanates significantly decreased cell viability and inhibited migration of populations consisting of different amounts of drug-sensitive and drug-resistant cells. In case of population entirely composed of lapatinib-resistant cells the most
\end{abstract}

Anna Herman-Antosiewicz

anna.herman-antosiewicz@biol.ug.edu.pl

1 Department of Molecular Biology, Faculty of Biology, University of Gdańsk, Wita Stwosza 59, 80-308 Gdańsk, Poland effective was combination of lapatinib with erucin which decreased cell viability and motility, phosphorylation of Akt, S6 and VEGF level more efficiently than each agent alone.

Conclusions Combination of lapatinib and isothiocyanates, especially erucin, might be considered as an effective treatment reducing metastatic potential of breast cancer cells, even these with the drug resistance phenotype.

Keywords HER2 - Isothiocyanates · Erucin - Lapatinib resistance

\section{Introduction}

Overproduction of HER2 (human epidermal growth factor receptor 2) is reported in nearly $20-25 \%$ of all breast cancer cases. Enhanced signal transduction from amplified receptors, especially through Akt-mTOR-S6K signaling pathway, leads to uncontrolled proliferation, evasion of apoptosis, neoangiogenesis and enhanced cell motility, which may lead to metastasis. It has been reported that $30-40 \%$ of women with breast cancer develop a metastatic disease [1], and that it may be associated with $H E R 2$ gene amplification [2]. Since overproduction of HER2 correlates with poor clinical outcomes, this receptor became a target for anticancer therapies.

Currently, several treatments targeting HER2 are approved, which include monoclonal antibodies and small molecule inhibitors of receptor kinases or their combinations with chemotherapeutic agents [3, 4]. Trastuzumab (Herceptin; Genentech, South San Francisco, CA, USA) was the first monoclonal antibody developed to target HER2. It significantly improved outcomes for patients diagnosed with this subtype of cancer [5]. However, de 
novo or acquired resistance to trastuzumab eventually occurs in most patients with advanced disease [6]. Thus, trastuzumab is typically combined with chemotherapy to increase efficacy, which also increases toxicity. Additional HER2 targeting agents have been developed recently, such as pertuzumab or trastuzumab emtansine. Pertuzumab (Perjeta, Genentech) is a fully humanized monoclonal antibody that binds to a different epitope of the HER2 extracellular domain than trastuzumab, and prevents dimerization of the HER receptors [7]. It is effective in combination with trastuzumab and docetaxel in advanced breast cancers [8]. In 2013, the FDA approved the first successful HER2-targeted antibody-drug conjugate, trastuzumab emtansine (T-DM1; Kadcyla; Genentech), for the treatment of HER2-positive trastuzumab-pretreated advanced breast cancer. This drug inhibits HER2 signaling and is cytotoxic to HER2-positive cells due to emtansine which disrupts dynamics of microtubules [9]. T-DM1 appears to have some activity against central nervous system metastases [10].

Another class of agents used to block Her-2 signaling are small molecule tyrosine kinase inhibitors, such as lapatinib (Tykerb; GlaxoSmithKline, Brentford, United Kingdom). Mechanism of its action relies on blocking of the ATP-binding site in the cytoplasmic domain of HER2 which leads to inhibition of signal transduction cascade from the receptor [4]. In spite of a great success in breast cancer therapy, primary or acquired resistance to lapatinib still occurs, even when this medicament is used in combination with other commercially available anti-HER2 agents (e.g., trastuzumab) [11]. Long-term exposure to lapatinib causes elimination of drug-sensitive cells and at the same time increases probability of selection of lapatinib-resistant cells whose percentage increases in the cell population with time. Cancer cells respond to the first stage of treatment and tumor decreases, however, appearance of resistant cells, which typically occurs within 12 months of the start of therapy, may lead to progression of the disease and to metastasis [12, 13]. Many different mechanisms underlying this phenomenon have been proposed, including hyperactivation of the signaling network downstream of HER2 [14].

Overexpression of HER2 is also associated with vascular endothelial growth factor (VEGF) upregulation [15]. It has been shown that most types of human cancer cells overexpress VEGF and its receptor [16]. VEGF is one of the most important factors stimulating angiogenesis, which is essential for the growth of solid tumors. VEGF also induces expression of matrix metalloproteinases (MMPs) that degrade the basement membrane, thus it is also involved in first stages of endothelial cell migration and metastasis of cancer cells [17].
Recently, we demonstrated that isothiocyantes, such as sulforaphane (SFN), erucin (ERN) and sulforaphene (SF), enhance anti proliferative activity of lapatinib in HER2positive breast cancer cells which was connected with a more efficient inhibition of pro survival signaling and induction of apoptosis [18]. Isothiocyanates (ITC) are naturally occurring compounds in Brassicaceae vegetables. They possess chemopreventive activities_-inhibit phase I enzymes, which are responsible for carcinogens activation, and induce phase II enzymes that are involved in carcinogen elimination [19, 20]. Moreover, isothiocyanates reveal anticancer activity causing apoptosis through induction of oxidative stress and modulation of numerous cell signaling cascades which are crucial for cancer cell survival [21]. It has been previously shown that SFN inhibits Akt-mTOR survival pathway in leukemia cells [22] and in breast cancer cells differing in growth factor receptor status [23]. Importantly, there are reports showing that SFN more efficiently inhibits growth of human breast cancer cells than normal breast epithelial cells [24, 25].

Numerous reports indicate that isothiocyanates influence different signal transduction pathways downstream of growth factor receptors. For instance, Wu et al. suggested that phenethyl isothiocyanate (PEITC) and benzyl isothiocyanate (BITC) inhibited cell survival signaling kinase Akt, and suppressed lung cancer cell metastasis potential [26]. SFN, one of the best characterized ITC present in high concentrations in broccoli, decreased phosphorylation of Akt and S6K1 in MDA-MB-231, MCF-7, MDA-MB468 and SKBR-3 breast cancer cell lines [23] and inhibited Akt and mTOR pathway in acute lymphoblastic leukemia cells [22]. Moreover, in PC-3 prostate cancer cells, SFN inhibited activation of NF- $\kappa \mathrm{B}, \mathrm{Akt}$ and ERK which are all involved in cancer survival and metastasis [27]. Furthermore, in vivo, SFN inhibited the activation of MMPs and lung metastasis induced by melanoma cells in mice [28]. It has been also shown that SFN suppressed capillary-like tube formation on basement membrane matrix and inhibited HMEC-1 cell migration by down regulation of the vascular endothelial growth factor (VEGF) and its receptor KDR/flk-1, hypoxia-inducible factor- $1 \alpha$, c-Myc and matrix metalloproteinase 2 [29].

In this work we asked a question whether combination of lapatinib with sulforaphane, sulphoraphene or erucin overcomes development of the drug-resistant populations and whether such therapy protects against development of metastatic breast cancer. We have chosen lapatinib, because - in contrast to trastuzumab and its derivatives-it is orally bioavailable drug. Additionally, as a small molecule it is better suited to cross the blood-brain barrier, which rationalizes its use in patients with metastases to central nervous system for which HER2-positive tumors have predilection [30]. 
To answer these questions, we designed an in vitro simulation of the drug-dependent selection of resistant cells during lapatinib treatment and evaluated anticancer efficiency of combined therapy as compared to lapatinib or each of ITCs as the only agents. Lapatinib-sensitive and lapatinib-resistant SKBR-3 cells were combined in different proportions to simulate the heterogeneity of cancer cell populations and were treated with low doses of isothiocyanates (close to their $\mathrm{IC}_{50}$ ), the drug (below its $\mathrm{IC}_{50}$ for the sensitive cell line) or combinations of compounds. Their survival and migration potential was evaluated. As erucin-lapatinib combination was the most effective, we verified its influence on cell motility, invasion, VEGF level and HER2 downstream signaling.

\section{Materials and methods}

\section{Reagents and cell lines}

Lapatinib ditosylate (purity $>99.5 \%$ ), erucin (purity $\geq 99 \%$ ), S-sulforaphene (purity $\geq 99.7 \%$ ) and R,Ssulforaphane (purity $\geq 99 \%$ ) were obtained from LKT Laboratories (St. Paul, MN, USA). DMSO, thiazolyl blue tetrazolium bromide (MTT), the anti- $\beta$-actin, anti-mouse and anti-rabbit antibodies conjugated with HRP were from Sigma (St. Louis, MO, USA). The antibodies against p-Akt (Ser-473), Akt, p-S6 (Ser 235), PARP-1, VEGF from Santa Cruz Biotechnology (Santa Cruz, CA, USA) and p-HER2 (Tyr121/1222), HER2, S6 were from Cell Signaling Technology (Danvers, MA, USA).

Lapatinib-resistant SKBR-3 cells (LapR) were developed by a long-term exposure of SKBR-3 cells to increasing concentrations of the drug. Cells were cultured in RPMI 1640 medium supplemented with $10 \%$ fetal bovine serum and $1 \%$ penicillin/streptomycin mixture (Life Technologies, Carlsbad, CA, USA). The invasive potential of the cells was determined using BD BioCoat ${ }^{\mathrm{TM}}$ BD MatrigelTM Invasion Chamber (BD Biosciences, MA, USA).

\section{Cell viability assay}

Lapatinib-sensitive and lapatinib-resistant cells were mixed in different proportions (percentage of resistant cells per probe was: $0 ; 5 ; 10 ; 25 ; 50 ; 75$ or $100 \%$ ). Combinations of SKBR-3 cells and their lapatinib-resistant counterparts (total concentration: $2 \times 10^{3}$ per well in $100 \mu \mathrm{l}$ of medium) were seeded into 96 -well plate and incubated at $37{ }^{\circ} \mathrm{C}$ and $5 \% \mathrm{CO}_{2}$ for $24 \mathrm{~h}$. After that time, the medium was removed and fresh medium containing either lapatinib (100 nM), one of ITCs $(2.5 \mu \mathrm{M}$ sulforaphene, $5 \mu \mathrm{M}$ sulforaphane or $5 \mu \mathrm{M}$ erucin) or combinations of compounds were added. After $48 \mathrm{~h}$ cells were incubated with $26 \mu \mathrm{l}$ of MTT ( $4 \mathrm{mg} / \mathrm{ml}$ ) for additional $3 \mathrm{~h}$. Then, the medium was removed and formazan crystals were dissolved in $100 \mu \mathrm{l}$ of DMSO. Absorbance (570 and $660 \mathrm{~nm}$ ) was measured in Victor3 Multilabel Counter (PerkinElmer, USA). Each dose of ITC and lapatinib was tested in triplicate and the experiment was repeated twice.

\section{Western blotting}

Drug-sensitive and drug-resistant SKBR-3 cells were mixed in different proportions and seeded in $4 \mathrm{ml}$ of medium in $6-\mathrm{cm}$ plates. After $24 \mathrm{~h}$, the medium was removed and replaced with a new one, containing lapatinib (100 $\mathrm{nM})$, erucin $(5 \mu \mathrm{M})$ or combination of compounds. After $48 \mathrm{~h}$, cells were collected and cell lysates, SDSPAGE electrophoresis and immunoblotting were performed as described previously [18]. To estimate the intracellular level of VEGF protein, cells were seeded in $8 \mathrm{ml}$ of medium in 10-cm plates and allowed to attach for 24-h and treated with erucin, the drug or their combination for $96 \mathrm{~h}$. The intensity of the immunoreactive bands was determined by densitometric scanning to quantify changes in protein levels. Each protein was detected at least twice, from independently prepared lysates.

\section{Migration assay}

$1 \times 10^{6}$ cells were seeded in $4 \mathrm{ml}$ of medium in $6 \mathrm{~cm}$ plates and a wound was made after 3 days, when cells achieved $100 \%$ confluence as described by Burk [31]. Cells were washed twice with PBS and a fresh medium containing $5 \mu \mathrm{M}$ erucin and/or $100 \mathrm{nM}$ lapatinib was added. After incubation for 7 days (the medium was exchanged every 2 days) cells were photographed using a light microscope. Experiment was performed three times. Migration was quantified by measuring the distance that cells migrated from the wound edge, at three positions of each photo taken from five randomly selected fields.

\section{Invasion assay}

The effect of erucin-lapatinib treatment, as well as each of this agent alone, on in vitro invasion was determined using BD BioCoat ${ }^{\mathrm{TM}}$ BD MatrigelTM Invasion Chamber according to the manufacturer's instructions. Briefly, after rehydratation of the chambers with serum-free medium for $2 \mathrm{~h}$ at $37{ }^{\circ} \mathrm{C}, 6 \times 10^{4}$ cells were seeded in serum-free medium supplemented with the tested agents or DMSO in each matrigel-coated insert containing membrane with an $8 \mu \mathrm{m}$ pore size. The lower compartment of the chamber contained $0.75 \mathrm{ml}$ of medium with chemoattractant $(10 \%$ FBS). After $48 \mathrm{~h}$, matrigel and non invading cells were 
removed; invading cells were fixed with $100 \%$ methanol, stained with crystal violet and counted under light microscope. Experiment was performed in triplicate.

\section{Statistical analysis}

Data were analyzed using GraphPad Prism software. Differences between groups in migration and invasion tests were analyzed using Student's $t$ test. Difference was considered significant at $P<0.05$. Differences between groups in viability tests were analyzed using two-way ANOVA followed by Bonferroni's multiple comparison test.

\section{Results}

\section{The effect of lapatinib, isothiocyanates and their combinations on viability of drug-sensitive and drug-resistant SKBR-3 cells}

To investigate the influence of combined treatment on viability of cell populations consisting of different proportions of lapatinib-sensitive and lapatinib-resistant cells we used the MTT assay. We chose low doses of isothiocyanates (close to their $\mathrm{IC}_{50}$ ) and the drug (below $\mathrm{IC}_{50}$ for the sensitive cell line) in combinations, which have been shown previously to act in a synergistic way [18]. Our model showed that in populations with high percentage of sensitive cells lapatinib was almost as effective as a low dose of an isothiocyanate, however, combined therapy was the most effective (Fig. 1). When percentage of resistant cells increased, lapatinib alone was less effective but low viability level was sustained due to isothiocyanates activity, either alone or in combination with lapatinib. Combination of lapatinib and erucin was the most efficient in cancer cell viability inhibition, even in the drug-resistant population (Fig. 1b).

\section{Apoptosis induction and cell signaling analysis upon lapatinib-erucin treatment}

To get insight into the mechanism involved in lapatinib resistance acquisition we compared status of crucial members of HER2 signaling pathway in non treated drugsensitive or drug-resistant SKBR-3 cells. As shown in Fig. 2a, in lapatinib-resistant cells the basal level of p-HER2 was much lower than in sensitive cells while the total level of the receptor was similar. The main HER2 downstream effector, the Akt kinase, was slightly decreased in populations enriched in lapatinib-resistant cells. On the other hand, the phosphorylation of ribosomal protein S6, substrate of mTOR-S6K1 was significantly lower in the drug resistant population which was accompanied by a drop in a total level of this protein (Fig. 2a).

To determinate the mechanism of anti-proliferative activity of the combination treatment we used the most effective pair, lapatinib and erucin, and looked at their effect on the apoptosis induction (PARP cleavage) and signal transduction pathway downstream of HER2 (p-Akt and p-S6). In the case of samples exclusively consisting of sensitive cells, we observed the highest percentage of cleaved PARP when cells were exposed to combined therapy, as compared to those treated either with erucin or lapatinib alone which is consistent with previously published results [18]. When the percentage of resistant cells was increased, PARP cleavage was induced mostly due to erucin activity (Fig. 2b).

The drop in the level of phosphorylated Akt kinase was comparable between erucin and erucin with lapatinibtreated cells, although in case of fully drug-resistant cells combination of compounds more efficiently decreased p-Akt than single compounds (Fig. 2b). We also observed that in the case of sensitive cells, the level of the phosphorylated ribosomal protein $\mathrm{S} 6$ (p-S6) was reduced by both erucin and the drug, and although in other samples, where proportion of sensitive to resistant cells was 3:1, 1:1 or $0: 1$, lapatinib or erucin activities were lower, combined treatment still caused the most effective decrease of p-S6 level comparing with effects of single compounds (Fig. 2b).

\section{Inhibition of motility and invasion of the drug- sensitive and drug-resistant SKBR-3 cells by the combination of erucin and lapatinib}

As Akt-mTOR pathway regulates cancer cell migration and invasion, we investigated whether the combined treatment impacts cell motility using wound-healing assay. Effect of lapatinib, at used by us concentration, on cell migration was weak, irrespective of percentage of the drug resistant cells in the population. However, we noticed that combinations of lapatinib with sulforaphane, erucin or sulforaphene significantly inhibited cell migration as compared to activity of each agent alone (data not shown). We observed the most effective cell motility inhibition for the combination of lapatinib with erucin, both in lapatinibsensitive (Fig. 3a) and lapatinib-resistant (Fig. 3b) SKBR3 cells.

To evaluate the invasive potential of the drug-sensitive and the drug-resistant SKBR-3 breast cancer cells in response to erucin, lapatinib and combined treatment, we used a transwell matrigel invasion assay. We determined, that combined treatment most efficiently decreased invasiveness of both tested cell lines, as compared to the effect of erucin and the drug alone, however, statistical 


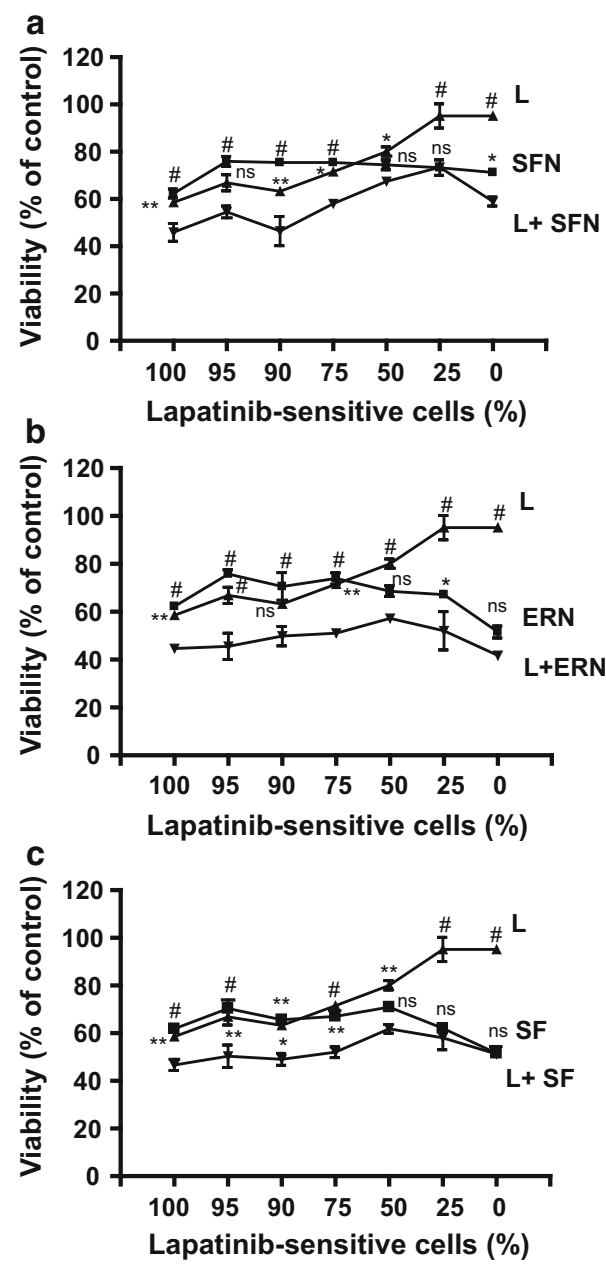

Fig. 1 Isothiocyanates sensitize SKBR-3 cells to lapatinib. Lapatinib-sensitive cells were mixed with lapatinib-resistant cells in various ratios (percentage of lapatinib-sensitive cells in a given sample is shown below the graph) and treated for $48 \mathrm{~h}$ with $5 \mu \mathrm{M}$ sulforaphane (SFN-a), $5 \mu \mathrm{M}$ erucin $(\mathrm{ERN}-\mathbf{b}), 2.5 \mu \mathrm{M}$ sulforaphene $(\mathrm{SF}-\mathbf{c}), 100 \mathrm{nM}$ lapatinib (L) or combinations. Cell viability was estimated by MTT assay. Each point is mean $( \pm \mathrm{SE})$ of two experiments done in triplicate (error bars are not shown when they are smaller than the symbols). Significant differences between single agent treatment and combination treatment for a given population are indicated as follows: $* P<0.05$; $* * P<0.01$; ${ }^{\#} P<0.001 ; n s$ not significant (two-way ANOVA followed by Bonferroni's multiple comparison test)

significance was achieved only in the case of the lapatinibsensitive cells (Fig. 4). In case of lapatinib-resistant cells, the invasiveness inhibition was caused mostly by erucin (Fig. 4b).

As VEGF is one of the most important regulators of angiogenesis, a complex process that may facilitate metastasis, we verified its level in cells treated with erucin, lapatinib or both agents. Our findings indicate that erucinlapatinib combination more efficiently decreased the VEGF level in drug-resistant than drug-sensitive cells (Fig. 4c).

\section{Discussion}

Lapatinib is one of the most efficient drugs against HER2positive breast cancer cells, and one of the most frequently used in therapy at the advanced, metastatic stage. By blocking intracellular ATP-binding site of the receptors, (HER2 and EGFR) lapatinib inhibits activation of both Ras/Raf/MEK/ERK and PI3K/Akt/mTOR/S6K signal transduction pathways, and consequently, induces cell cycle arrest in the G1 phase and apoptosis [32]. Lapatinib inhibits the receptor for a longer time in comparison to other anti-EGFR drugs, e.g., erlotinib (Tarceva) and gefitinib (Iressa), due to its long dissociation half life that is more than $300 \mathrm{~min}$. This drug shows activity in case of breast cancer cell lines that are resistant to trastuzumab. Lapatinib decreases activation of S6K and IGF-1, two signaling pathways that are considered as mediators in trastuzumab resistance [4].

Despite the great success of lapatinib, its use in therapy often becomes ineffective and after incipient tumor regression, recurrence of the disease is observed. This progression is due to primary or acquired resistance [12]. During longterm lapatinib therapy, on the one hand drug-sensitive cells are eliminated; on the other hand probability of drug-resistant cell selection increases [13]. Several mechanisms of acquisition of resistance to lapatinib have been proposed, e.g., mutations within genes of HER2 or EGFR receptors, increased signal transduction from estrogen receptor, overexpression of progesterone receptor, hyperactivation of PIK3Ca or mutations in genes of the PI3K pathway [11, 12]. Lapatinib also derepresses FOXO3a, which stimulates transcription of estrogen receptor (ER) and leads to co-dependence on HER2 and ER signaling [33].

One of the potential ways to overcome drug resistance is a therapy that uses combination of several anti-HER2 drugs, nonetheless lapatinib-based therapy has adverse events (rash, diarrhea, cold symptoms, vomiting, anorexia, nausea, fatigue, headache, gastrointestinal symptoms and hepatotoxicity) $[4,34]$. This problem is even more serious in case of treatment using two or more drugs. For example, preclinical studies have shown that combination of lapatinib with trastuzumab more efficiently downregulates survivin and induces apoptosis, than each of these agents alone. However, trastuzumab may impair cardiac function by causing myocardium damage and despite the fact that the mechanism of this cardiac dysfunction is not known, it has been suggested that HER2 may play a protective role for cardiomyocytes [35]. Thus, side effects are the main limitation of such combined therapy.

In this work, we developed cell-based model of lapatinib resistance using HER2- overproducing SKBR-3 cells that were cultured for a few months in the presence of 
a

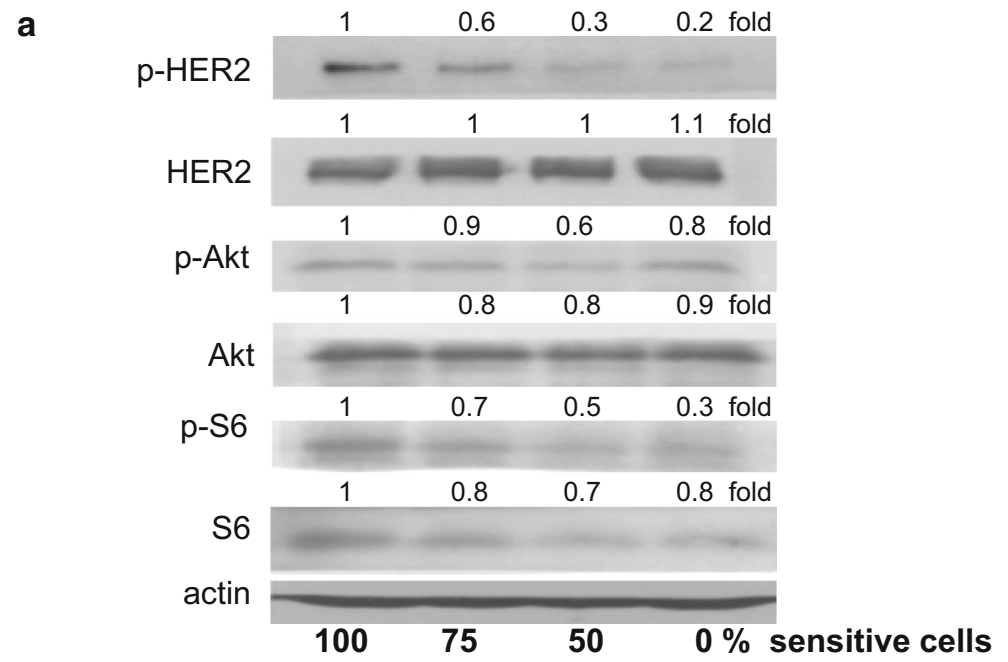

b

S:R (\%)

100:0

$75: 25$

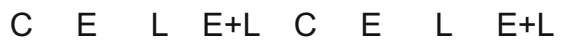
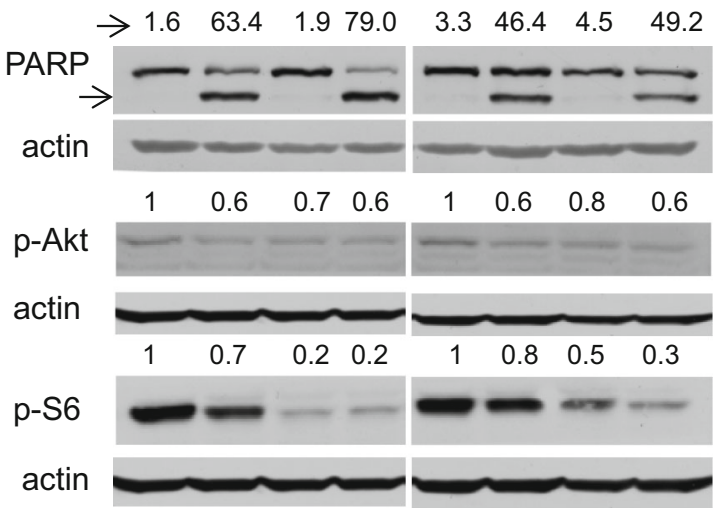

Fig. 2 a Comparison of the levels and phosphorylation of HER2, Akt and S6 in populations of lapatinib-sensitive and/or lapatinib-resistant cells. b Impact of erucin, lapatinib or the combination on PARP cleavage as well as phosphorylation of Akt and ribosomal S6 in cell populations composed of lapatinib-sensitive and lapatinib-resistant SKBR-3 cells. Lapatinib-sensitive and lapatinib-resistant cells mixed in ratio shown above blots $(\mathrm{S}: \mathrm{R})$ were treated with $5 \mu \mathrm{M}$ erucin $(\mathrm{E})$,

increasing drug concentrations. IC50 for the parental cell line was $120 \mathrm{nM}$, while their lapatinib-resistant derivatives were able to grow in the presence of more than $600 \mathrm{nM}$ drug. Resistance was not associated with the loss of HER2 expression or sensitivity to lapatinib because phosphorylation of its downstream effectors, such as Akt, was still inhibited in resistant cells. It indicates that resistance occurred by an alternative pathway, similarly as has been previously observed in the case of lapatinib-resistant BT474 cells [36].

We further worked on mixed cell populations consisting of the drug-sensitive and the drug-resistant cancer cells in various proportions which might mimic heterogeneity of breast tumor in vivo in context of the resistance acquisition

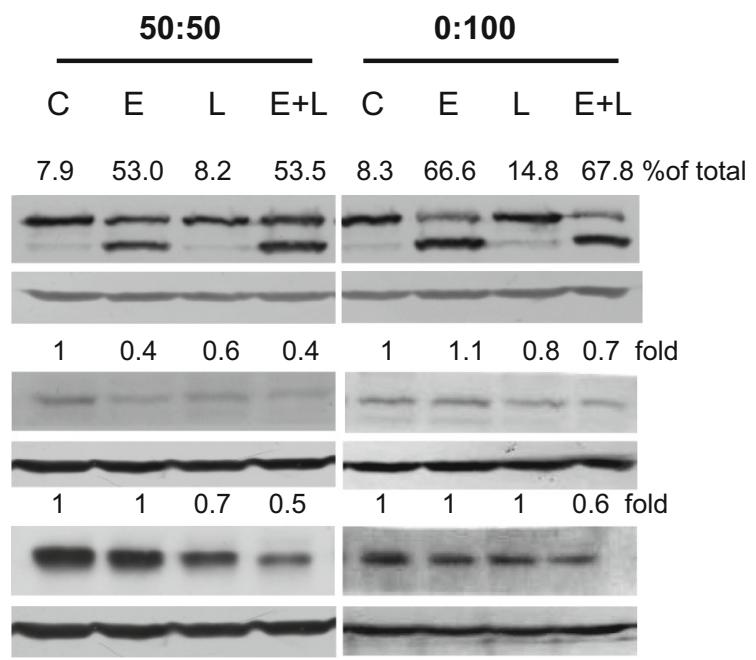

$100 \mathrm{nM}$ lapatinib (L) or combination of both compounds for $48 \mathrm{~h}$. Blots were stripped and reprobed with anti- $\beta$-actin antibody to verify equal protein loading. Densitometric analysis data after correction for loading control and relative to control are on top of the respective bands. In case of PARP, the percentage of cleaved band in the whole amount of PARP is shown. Similar results were observed in replicate experiments

process. We tested efficiency of anticancer activity of lapatinib, one of isothiocyanates (sulforaphane, erucin or sulforaphene) and their combinations applied in relatively low concentrations which have been previously shown to work in a synergistic way and inhibit downstream elements of the HER-2 pathway in lapatinib-sensitive cells [18]. Our findings support hypothesis that isothiocyanates sensitize lapatinib-resistant cells to the drug. Even when percentage of the resistant cells increased, and drug sensitivity decreased, combination of lapatinib with any of the isothiocyanates tested caused a decline in cell viability and migration potential, as compared to activity of each agent alone.

Interestingly, relatively poorly known erucin in combination with lapatinib was more potent that other 
a
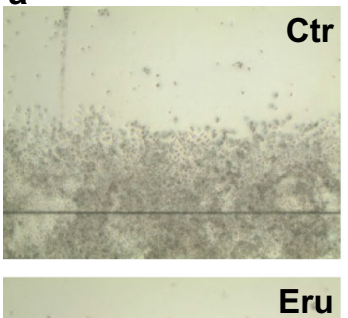

Eru

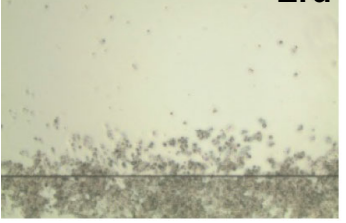

b

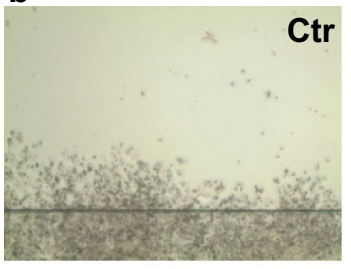

Eru
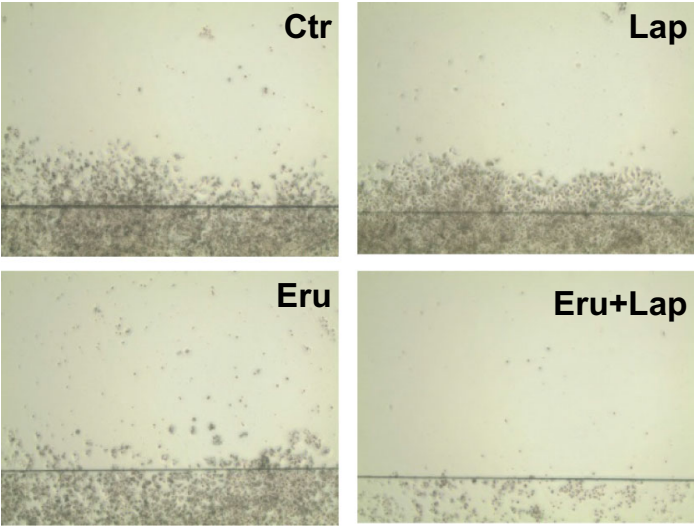

Eru+Lap

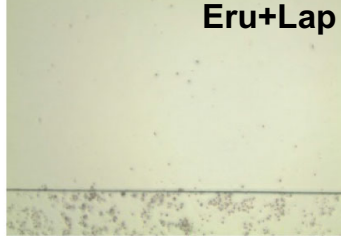

Fig. 3 Combination of lapatinib with erucin significantly inhibits cell migration as compared to activity of each agent alone. Confluent monolayers of lapatinib-sensitive (a) or lapatinib-resistant (b) SKBR3 cells were wounded with a razor blade. After 7 days, migration

combinations. Thus, molecular mechanism was investigated in more details for this combination. The apoptosis marker, cleaved PARP, was increased in cells treated with lapatinib-erucin combination although when the percentage of resistant cells increased, erucin was mostly responsible for apoptosis induction. The analysis also revealed that the combination of lapatinib with erucin, similarly as in the drug-sensitive cells, downregulated PI3K-Akt-mTOR-S6K pathway, which was evidenced by a decrease in p-AKT and p-S6. This is an important observation as it has been reported that PI3K-Akt-mTOR-S6K1 signaling plays prominent roles in apoptosis suppression, drug resistance and metastasis. Alterations in this pathway in breast cancers are often caused by mutations or aberrant expression of numerous genes, including: HER2, BRCA1, BRCA2, EGFR1, ER $\alpha$, PTEN, PI3K, TP53, RB [37, 38]. S6K1 is one of the regulators of cancer cell invasion, migration and metastasis. It was demonstrated in a model of metastasis of triple-negative breast cancer that $\mathrm{S} 6 \mathrm{~K} 1$ promotes invasiveness [39]. Moreover, team led by Dihua Yu has shown that ErbB2 (HER-2) increases VEGF protein production by
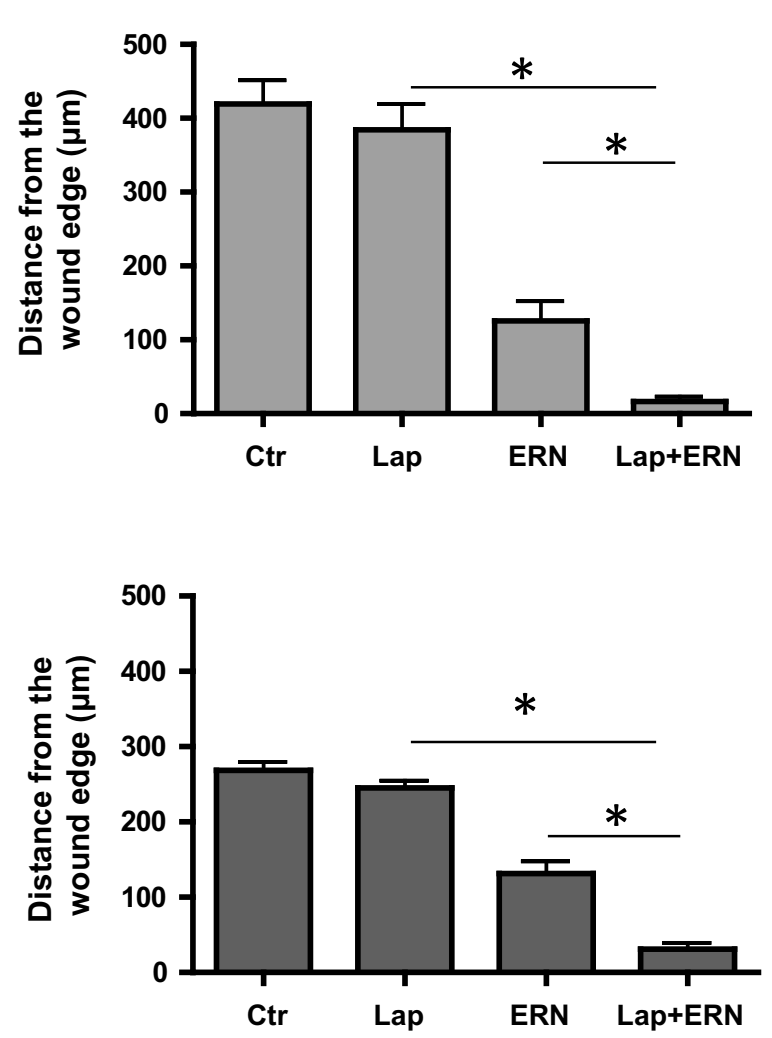

through the wound edge was examined under the microscope. Magnification $\times 4$. Data represent the mean \pm SE of three independent experiments, in each five randomly chosen fields were examined; $* P<0.05$

activating S6K in cell lines, xenografts and in human cancers [40]. This observation underscored suggestion that S6K activity may serve as a target for antiangiogenic and antimetastatic therapies [40]. In our model, combination of lapatinib with erucin inhibits S6K more efficiently than treatment with single agents, as revealed by a drop in phosphorylation of its main substrate, the ribosomal S6 protein, which might serve as a marker of successful treatment. Reduced activity of S6K might contribute to the observed decrease in intracellular VEGF level, as well as inhibition of motility and invasiveness of SKBR-3 cells. It is worth mentioning that the combined treatment effectively decreased VEGF and metastatic potential in drug-resistant cells, although effect on invasive potential was mostly exerted by erucin.

In conclusion, the obtained results suggest that application of isothiocyanates, especially erucin, as adjuvant agents during lapatinib therapy is reasonable. This novel solution may not only inhibit resistance acquisition and metastasis processes, but may also be less deleterious for patients as it uses a low dose of each agent. However, to 
a
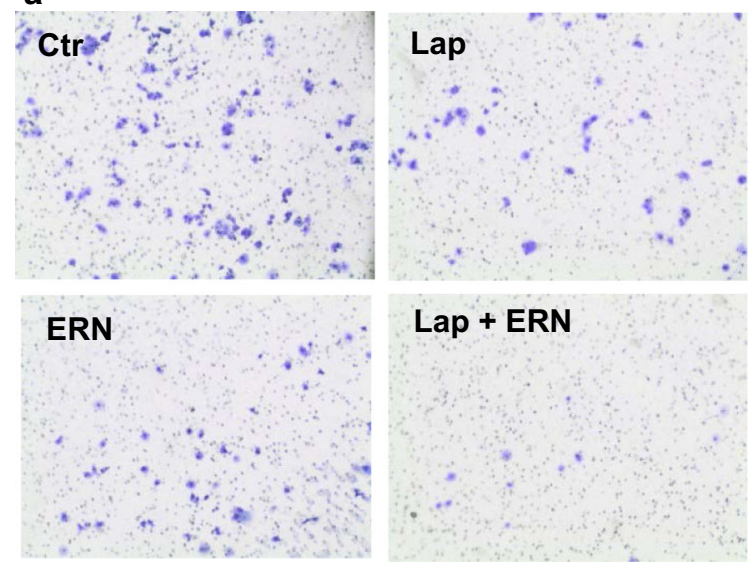

Lap + ERN

b
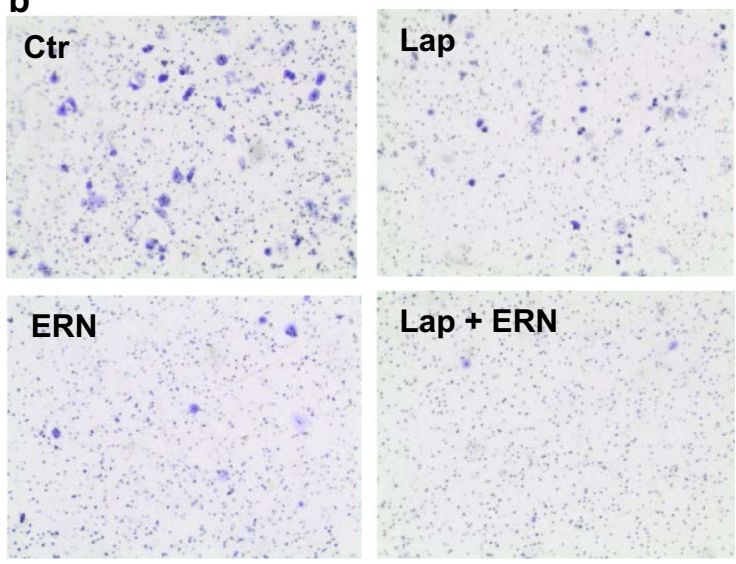

Lap + ERN
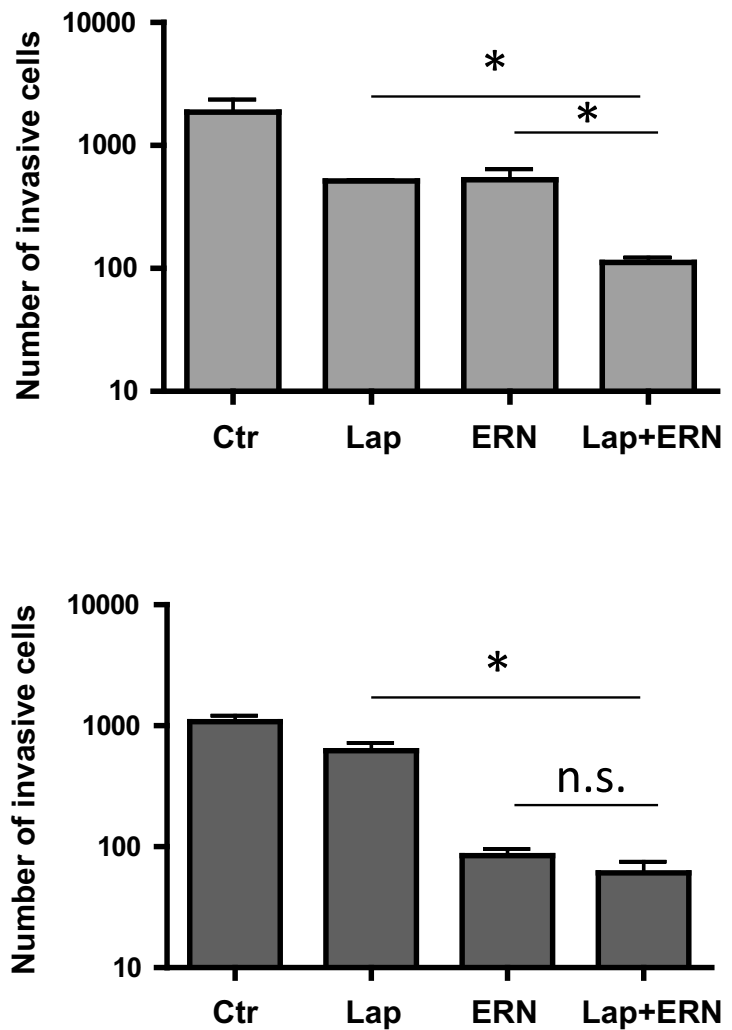

C

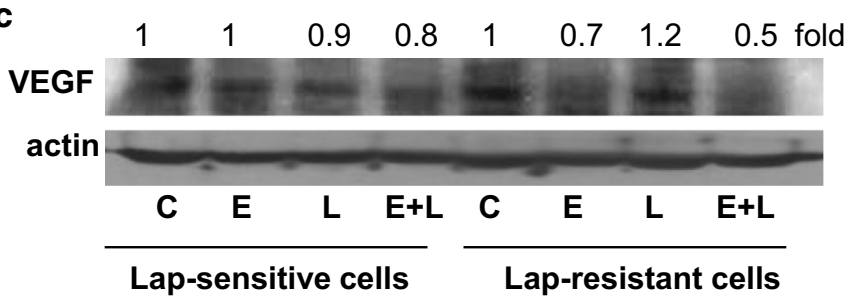

Fig. 4 Impact of mono- and combined treatment on invasion and VEGF level in SKBR-3 cells. Combination of lapatinib with erucin inhibits cell invasion of both, lapatinib-sensitive (a) and lapatinibresistant (b) SKBR-3 cells. Boyden chamber assay was performed after 48-h treatment, and invaded cells were counted. Data represent the mean $\pm \mathrm{SE}$ of three parallel experiments; $* P<0.05$, ns non

fully understand the potential of this method, further in vitro and in vivo experiments are required.

Acknowledgments We are grateful to Dr. K. Potrykus for language assistance. This investigation was supported by the National Science Centre (Poland), Project no. N N301 601740.

\section{Compliance with ethical standards}

Conflict of interest The authors declare that there are no conflicts of interest. significant. c VEGF level is efficiently reduced by combination of lapatinib with erucin. Cells were treated for $96 \mathrm{~h}$ with $5 \mu \mathrm{M}$ erucin (E), $100 \mathrm{nM}$ lapatinib (L) or combination of both compounds. Blots were stripped and reprobed with anti- $\beta$-actin antibody to ensure equal protein loading. Densitometric analysis data after correction for loading control and relative to respective controls are on top of bands

Open Access This article is distributed under the terms of the Creative Commons Attribution 4.0 International License (http://creative commons.org/licenses/by/4.0/), which permits unrestricted use, distribution, and reproduction in any medium, provided you give appropriate credit to the original author(s) and the source, provide a link to the Creative Commons license, and indicate if changes were made.

\section{References}

1. Da Silva L, Simpson PT, Smart CE, Cocciardi S, Waddell N, et al. HER3 and downstream pathways are involved in 
colonization of brain metastases from breast cancer. Breast Cancer Res. 2010;12:R46.

2. Hicks DG, Short SM, Prescott NL, Tarr SM, Coleman KA, et al. Breast cancers with brain metastases are more likely to be estrogen receptor negative, express the basal cytokeratin CK5/6, and overexpress HER2 or EGFR. Am J Surg Pathol. 2006;30:1097-104.

3. Wahler J, Suh N. Targeting HER2 positive breast cancer with chemopreventive agents. Curr Pharmacol Rep. 2015;1:324-35.

4. Rana P, Sridhar SS. Efficacy and tolerability of lapatinib in the management of breast cancer. Breast Cancer (Auckl). 2012;6:67-77.

5. Yin W, Jiang Y, Shen Z, Shao Z, Lu J. Trastuzumab in the adjuvant treatment of HER2-positive early breast cancer patients: a meta-analysis of published randomized controlled trials. PLoS One. 2011;6:e21030.

6. Montemurro F, Redana S, Viale G, Sanna G, Donadio M, et al. Retrospective evaluation of clinical outcomes in patients with HER2-positive advanced breast cancer progressing on trastuzumab-based therapy in the pre-lapatinib era. Clin Breast Cancer. 2008;8:436-42.

7. Franklin MC, Carey KD, Vajdos FF, Leahy DJ, de Vos AM, et al. Insights into ErbB signaling from the structure of the ErbB2pertuzumab complex. Cancer Cell. 2004;5:317-28.

8. Baselga J, Cortes J, Kim SB, Im SA, Hegg R, et al. Pertuzumab plus trastuzumab plus docetaxel for metastatic breast cancer. N Engl J Med. 2012;366:109-19.

9. Teicher BA, Doroshow JH. The promise of antibody-drug conjugates. N Engl J Med. 2012;367:1847-8.

10. Bartsch R, Berghoff AS, Vogl U, Rudas M, Bergen E, et al. Activity of T-DM1 in Her2-positive breast cancer brain metastases. Clin Exp Metastasis. 2015;32:729-37.

11. Wang YC, Morrison G, Gillihan R, Guo J, Ward RM, et al. Different mechanisms for resistance to trastuzumab versus lapatinib in HER2-positive breast cancers-role of estrogen receptor and HER2 reactivation. Breast Cancer Res. 2011;13:R121.

12. Chen FL, Xia W, Spector NL. Acquired resistance to small molecule ErbB2 tyrosine kinase inhibitors. Clin Cancer Res. 2008;14:6730-4.

13. Formisano L, Nappi L, Rosa R, Marciano R, D'Amato C, et al. Epidermal growth factor-receptor activation modulates Src-dependent resistance to lapatinib in breast cancer models. Breast Cancer Res. 2014;16:R45.

14. O'Brien NA, Browne BC, Chow L, Wang Y, Ginther C, et al. Activated phosphoinositide 3-kinase/AKT signaling confers resistance to trastuzumab but not lapatinib. Mol Cancer Ther. 2010;9:1489-502.

15. Falchook GS, Moulder SL, Wheler JJ, Jiang Y, Bastida CC, et al. Dual HER2 inhibition in combination with anti-VEGF treatment is active in heavily pretreated HER2-positive breast cancer. Ann Oncol. 2013;24:3004-11.

16. Grepin R, Pages G. Molecular mechanisms of resistance to tumour anti-angiogenic strategies. J Oncol. 2010;2010:835680.

17. Ghosh S, Basu M, Roy SS. ETS-1 protein regulates vascular endothelial growth factor-induced matrix metalloproteinase- 9 and matrix metalloproteinase-13 expression in human ovarian carcinoma cell line SKOV-3. J Biol Chem. 2012;287:15001-15.

18. Kaczyńska A, Świerczyńska J, Herman-Antosiewicz A. Sensitization of HER2 positive breast cancer cells to lapatinib using plants-derived isothiocyanates. Nutr Cancer. 2015;67:976-86.

19. Munday R, Munday CM. Induction of phase II detoxification enzymes in rats by plant-derived isothiocyanates: comparison of allyl isothiocyanate with sulforaphane and related compounds. J Agric Food Chem. 2004;52:1867-71.
20. Barcelo S, Gardiner JM, Gescher A, Chipman JK. CYP2E1mediated mechanism of anti-genotoxicity of the broccoli constituent sulforaphane. Carcinogenesis. 1996;17:277-82.

21. Gupta P, Kim B, Kim SH, Srivastava SK. Molecular targets of isothiocyanates in cancer: recent advances. Mol Nutr Food Res. 2014;58:1685-707.

22. Suppipat K, Park CS, Shen Y, Zhu X, Lacorazza HD. Sulforaphane induces cell cycle arrest and apoptosis in acute lymphoblastic leukemia cells. PLoS One. 2012;7:e51251.

23. Pawlik A, Wiczk A, Kaczyńska A, Antosiewicz J, Herman-Antosiewicz A. Sulforaphane inhibits growth of phenotypically different breast cancer cells. Eur J Nutr. 2013;52:1949-58.

24. Tseng E, Scott-Ramsay EA, Morris ME. Dietary organic isothiocyanates are cytotoxic in human breast cancer MCF-7 and mammary epithelial MCF-12A cell lines. Exp Biol Med (Maywood). 2004;229:835-42.

25. Sakao K, Singh SV. D, L-sulforaphane-induced apoptosis in human breast cancer cells is regulated by the adapter protein p66Shc. J Cell Biochem. 2012;113:599-610.

26. Wu X, Zhu Y, Yan H, Liu B, Li Y, et al. Isothiocyanates induce oxidative stress and suppress the metastasis potential of human non-small cell lung cancer cells. BMC Cancer. 2010;10:269.

27. Shankar S, Ganapathy S, Srivastava RK. Sulforaphane enhances the therapeutic potential of TRAIL in prostate cancer orthotopic model through regulation of apoptosis, metastasis, and angiogenesis. Clin Cancer Res. 2008;14:6855-66.

28. Gupta SC, Kim JH, Prasad S, Aggarwal BB. Regulation of survival, proliferation, invasion, angiogenesis, and metastasis of tumor cells through modulation of inflammatory pathways by nutraceuticals. Cancer Metastasis Rev. 2010;29:405-34.

29. Bertl E, Bartsch H, Gerhauser C. Inhibition of angiogenesis and endothelial cell functions are novel sulforaphane-mediated mechanisms in chemoprevention. Mol Cancer Ther. 2006;5:575-85.

30. Lin NU, Winer EP. Brain metastases: the HER2 paradigm. Clin Cancer Res. 2007;13:1648-55.

31. Burk RR. A factor from a transformed cell line that affects cell migration. Proc Natl Acad Sci USA. 1973;70:369-72.

32. Rusnak DW, Lackey K, Affleck K, Wood ER, Alligood KJ, et al. The effects of the novel, reversible epidermal growth factor receptor/ErbB-2 tyrosine kinase inhibitor, GW2016, on the growth of human normal and tumor-derived cell lines in vitro and in vivo. Mol Cancer Ther. 2001;1:85-94.

33. Leung WY, Roxanis I, Sheldon H, Buffa FM, Li JL, et al. Combining lapatinib and pertuzumab to overcome lapatinib resistance due to NRG1-mediated signalling in HER2-amplified breast cancer. Oncotarget. 2015;6:5678-94.

34. Nelson MH, Dolder CR. A review of lapatinib ditosylate in the treatment of refractory or advanced breast cancer. Ther Clin Risk Manag. 2007;3:665-73.

35. Mukohara T. Role of HER2-targeted agents in adjuvant treatment for breast cancer. Chemother Res Pract. 2011;2011:730360.

36. Xia W, Bacus S, Hegde P, Husain I, Strum J, et al. A model of acquired autoresistance to a potent ErbB2 tyrosine kinase inhibitor and a therapeutic strategy to prevent its onset in breast cancer. Proc Natl Acad Sci USA. 2006;103:7795-800.

37. Davis NM, Sokolosky M, Stadelman K, Abrams SL, Libra M, et al. Deregulation of the EGFR/PI3K/PTEN/Akt/mTORC1 pathway in breast cancer: possibilities for therapeutic intervention. Oncotarget. 2014;5:4603-50.

38. Ren W, Liu Y, Wan S, Fei C, Wang W, et al. BMP9 inhibits proliferation and metastasis of HER2-positive SK-BR-3 breast cancer cells through ERK1/2 and PI3K/AKT pathways. PLoS One. 2014;9:e96816. 
39. Khotskaya YB, Goverdhan A, Shen J, Ponz-Sarvise M, Chang SS, et al. S6K1 promotes invasiveness of breast cancer cells in a model of metastasis of triple-negative breast cancer. Am J Transl Res. 2014;6:361-76.

40. Klos KS, Wyszomierski SL, Sun M, Tan M, Zhou X, et al. ErbB2 increases vascular endothelial growth factor protein synthesis via activation of mammalian target of rapamycin/p70S6K leading to increased angiogenesis and spontaneous metastasis of human breast cancer cells. Cancer Res. 2006;66:2028-37. 